\title{
IUFOST2006/374
}

\section{Fate and impact of apple polyphenols during cider production}

\author{
C. Renard ${ }^{\mathrm{a}}$, S. Guyot ${ }^{\mathrm{a}}$, J.-M. Le Quéréa ${ }^{\text {, J. Primault }}{ }^{\mathrm{a}}$, A. Baron ${ }^{\mathrm{a}}$, R. Bauduin ${ }^{\mathrm{a}}$, C. Le Bourvellec ${ }^{\mathrm{a}}$, S. \\ Bernillon $^{\mathrm{a}}$, A. Nogueira ${ }^{\mathrm{a}}$, J. Pagès $^{\mathrm{b}}$ and J.-F. Drilleau ${ }^{\mathrm{a}}$ \\ ${ }^{a}$ INRA - Research Unit : Cider Production, Fruit \& Vegetable Bioprocessing, Domaine de la motte, 35653 \\ Le Rheu, France \\ bLaboratoire de Mathématiques Appliquées, Agrocampus, 35000 Rennes, France \\ catherine.renard@rennes.inra.fr
}

Polyphenols influence the organoleptic properties of cider. Polyphenols in cider apples are those of table apples but with higher concentrations. The three main classes are flavan-3-ols, monomeric (catechins) and oligomeric or polymeric (procyanidins), hydroxycinnamic acids and dihydrochalcones. Procyanidins generate bitterness and/or astringency, depending on their size. The colour of ciders is due to " enzymatic browning ", a complex chain of reactions initiated by the oxidation of cafeoylquinic acid onto its highly reactive quinone by polyphenoloxydase.

Apple phenolics were followed quantitatively during cider elaboration for five varieties during three years and at two maturation steps. Their impact on the taste and mouthfeel of the final cider were assessed. Three points were studied in depth : - retention of tannins on apple pomace; - evolution of the quinones generated by action of polyphenoloxydase on cafeoylquinic acid ; - competition for dioxygen between yeasts and polyphenoloxydase.

Major differences occurred between fruit and must. Fermentation by Saccharomyces uvarum had very little impact on the polyphenolic composition. Organoleptic assessment of the final ciders confirmed the link between polyphenols and bitterness and/or astringency; the jury detected differences due to post-harvest maturation and pomace oxigenation prior to pressing. Isolated procyanidins bound spontaneously to apple cell walls by H-bonds and hydrophobic interactions. The amount of bound procyanidins increased with their size and concentration, and could be influenced by temperature, presence of salts or ethanol, and by the physical state of the cell walls. These interactions explain the selective retention of high molecular weight procyanidins during apple pressing. Oxidation products were systematically searched in apple musts by LCMS. Numerous products were present, and we identified addition products involving molecules of the same class, sometimes with addition of water, and co-dimers i.e. from reaction between different classes. Four dimers of cafeoylquinic acid were isolated from synthetic solutions and their structures were elucidated by LC-MSn and NMR. Polyphenoloxydase activity and polyphenol concentrations in cider apple juices are such that all dissolved oxygen is rapidly consumed. Anaerobic conditions are thus established at the start of fermentation, and will influence yeast growthand indirectly the stability of the final product.

The phenolic composition of cider is for the most part established at pressing, and we have identified a key role of oxygen in cider fermentations. More work is needed notably to further clarify the diversity of oxidation products, their reaction schemes and organoleptic properties. 